\title{
Causal effects of atrial fibrillation on brain white and gray matter volume: a Mendelian randomization study
}

Sehoon Park ${ }^{1,2}$, Soojin Lee ${ }^{3,4,5}$, Yaerim Kim ${ }^{6}$, Semin Cho ${ }^{3,4}$, Kwangsoo Kim, Yong Chul Kim³ , Seung Seok Han, Hajeong Lee ${ }^{3}$, Jung Pyo Lee ${ }^{3,8,9}$, Soryoung Lee ${ }^{3,4}$, Eue-Keun Choi ${ }^{3,4}$, Kwon Wook Joo ${ }^{3,4,8}$, Chun Soo Lim³, ${ }^{3,8,9}$, Yon Su Kim ${ }^{1,3,4,8}$ and Dong Ki Kim ${ }^{3,4,8^{*}}$

\begin{abstract}
Background: Atrial fibrillation (AF) and brain volume loss are prevalent in older individuals. We aimed to assess the causal effect of atrial fibrillation on brain volume phenotypes by Mendelian randomization (MR) analysis.

Methods: The genetic instrument for AF was constructed from a previous genome-wide association study (GWAS) meta-analysis (15,993 AF patients and 113,719 controls of European ancestry). The outcome summary statistics for head-size-normalized white or gray matter volume measured by magnetic resonance imaging were provided by a previous GWAS of 33,224 white British participants in the UK Biobank. Two-sample MR by the inverse varianceweighted method was performed, supported by pleiotropy-robust MR sensitivity analysis. The causal estimates for the effect of AF on ischemic stroke were also investigated in a dataset that included the findings from the MEGASTROKE study (34,217 stroke patients and 406,111 controls of European ancestry). The direct effects of AF on brain volume phenotypes adjusted for the mediating effect of ischemic stroke were studied by multivariable MR.

Results: A higher genetic predisposition for AF was significantly associated with lower grey matter volume [beta -0.040 , standard error (SE) $0.017, P=0.017$ ], supported by pleiotropy-robust MR sensitivity analysis. Significant causal estimates were identified for the effect of AF on ischemic stroke (beta 0.188, SE 0.026, $P=1.03 E-12$ ). The total effect of AF on lower brain grey matter volume was attenuated by adjusting for the effect of ischemic stroke (direct effects, beta -0.022 , SE $0.033, P=0.528$ ), suggesting that ischemic stroke is a mediator of the identified causal pathway. The causal estimates were nonsignificant for effects on brain white matter volume as an outcome.
\end{abstract}

Conclusions: This study identified that genetic predisposition for AF is significantly associated with lower gray matter volume but not white matter volume. The results indicated that the identified total effect of AF on gray matter volume may be mediated by ischemic stroke.

Keywords: Atrial fibrillation, Brain, Stroke, Mendelian randomization

\footnotetext{
* Correspondence: dkkim73@gmail.com

${ }^{3}$ Department of Internal Medicine, Department of Internal Medicine,

Uijeongbu Eulji University Medical Center, Gyeonggi-do, Uijeongbu, Korea

${ }^{4}$ Department of Internal Medicine, Seoul National University Hospital, 101

Daehak-ro, Jongno-gu, Seoul 03080, Korea

Full list of author information is available at the end of the article
}

(c) The Author(s). 2021 Open Access This article is licensed under a Creative Commons Attribution 4.0 International License, which permits use, sharing, adaptation, distribution and reproduction in any medium or format, as long as you give appropriate credit to the original author(s) and the source, provide a link to the Creative Commons licence, and indicate if changes were made. The images or other third party material in this article are included in the article's Creative Commons licence, unless indicated otherwise in a credit line to the material. If material is not included in the article's Creative Commons licence and your intended use is not permitted by statutory regulation or exceeds the permitted use, you will need to obtain permission directly from the copyright holder. To view a copy of this licence, visit http://creativecommons.org/licenses/by/4.0/ The Creative Commons Public Domain Dedication waiver (http://creativecommons.org/publicdomain/zero/1.0/) applies to the data made available in this article, unless otherwise stated in a credit line to the data. 


\section{Background}

Atrial fibrillation (AF) is the most common cardiac arrhythmia associated with the risk of stroke, heart failure, dementia, and mortality [1] and further contributes to a substantial socioeconomic burden [2]. The prevalence of AF is substantially increasing along with the global aging trend $[3,4]$.

As AF is highly prevalent in elderly individuals, cognitive dysfunction or functional brain disorders, which are also common in elderly people, have been associated with AF [5]. In addition, brain volume loss is related to persistent AF, along with low cerebral blood flow in AF patients [6-8]. However, demonstration of the causal effect of AF on structural brain volume changes has yet to be performed. Because pathologic brain volume loss and AF share risk factors and are both common in older individuals with multiple comorbidities, whether the observed low brain volume is a consequence of AF could hardly be answered by observational studies due to residual confounding effects. In addition, whether AF alone can cause brain volume loss even without stroke needs to be studied, as previous reports assessed the association between AF and dementia in stroke-free individuals, and brain volume loss was present before the identified first stroke event in $\operatorname{AF}$ patients $[5,9,10]$. Such evidence for the causal effect of AF on brain volume and its mechanistic association with stroke would suggest whether accelerated brain volume loss in AF patients may be ameliorated through appropriate AF management targeting the risk of ischemic stroke.

Mendelian randomization (MR) is an analytic method that can identify causal estimates with epidemiologic data [11]. MR utilizes a genetic instrument that is fixed before birth; thus, instrumented genetic predisposition is minimally affected by confounders or reverse causation. The significant association between genetic predisposition, which would result in a higher occurrence of the exposure of interest, and the outcome would suggest the presence of a causal effect of the exposure. MR has been widely introduced in the medical literature and has identified an important causal linkage between complex exposures and outcomes [12].

In this study, we performed a summary-level MR analysis to demonstrate the causal effects of AF on brain volume phenotypes. We also performed an MR analysis to investigate the causal pathway between AF, stroke, and brain white or gray matter volumes. We hypothesized that AF would decrease a certain type of brain volume that would be potentially mediated by ischemic stroke.

\section{Methods \\ Study setting}

The study was a summary-level MR analysis that mainly consisted of two parts (Fig. 1). The causal estimates for the effect of AF, genetically predicted by singlenucleotide polymorphisms (SNPs) reported in a previous

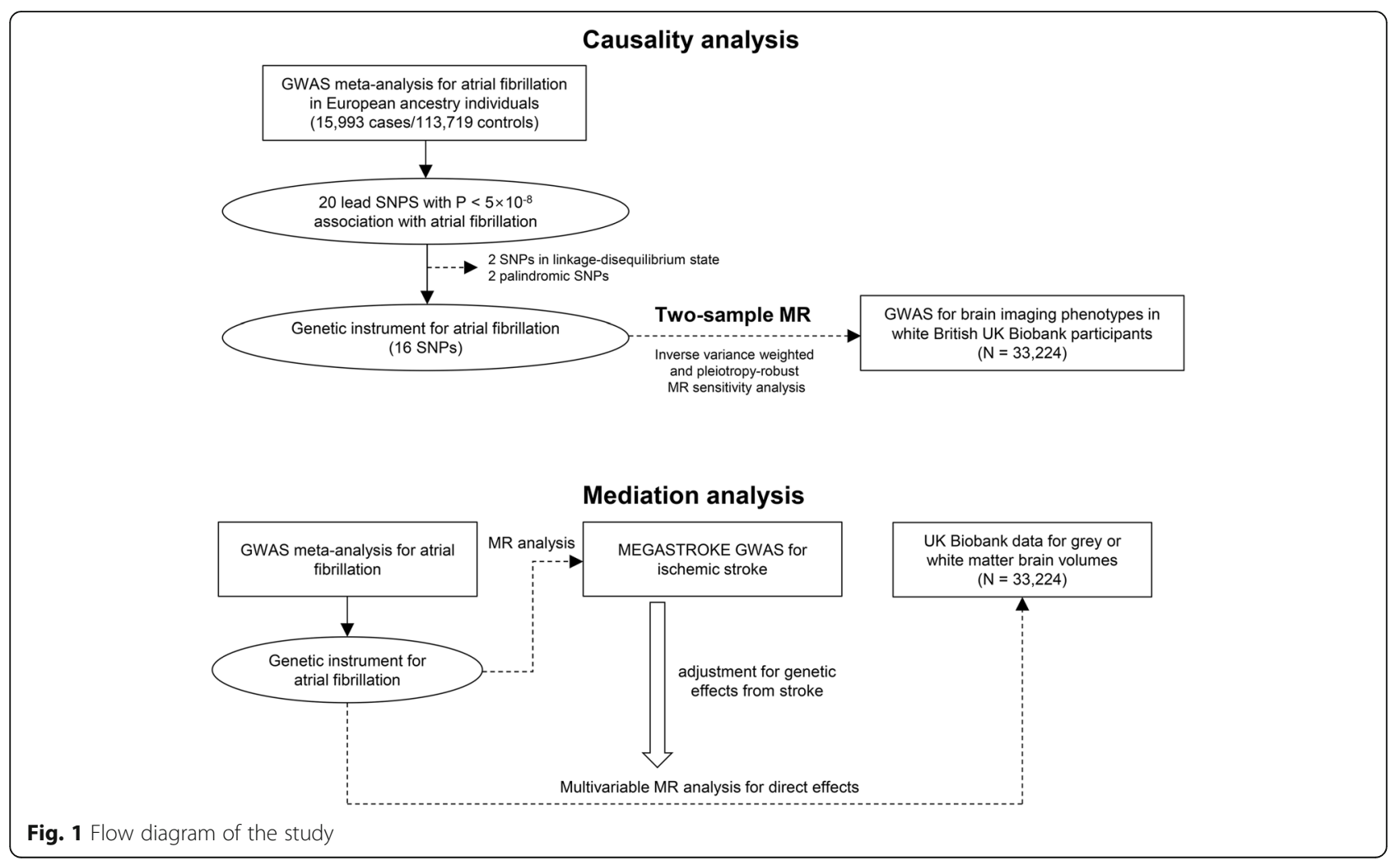


genome-wide association study (GWAS), on white or gray matter volume, measured in the independent UK Biobank data, were initially tested by two-sample MR. Next, additional GWAS results for ischemic stroke phenotype were utilized, and the causal effects of AF on ischemic stroke awere investigated. Finally, to determine whether the effects of AF on brain volume are mediated by ischemic stroke, multivariable MR analysis adjusted for the genetic effects of ischemic stroke was performed.

\section{Genetic instrument for AF}

Data from a previous GWAS meta-analysis for AF were used for this study $[13,14]$. Unlike from our previous study also investigating the causal estimates from AF $[12,15]$, as the outcome data solely consisted of the UK Biobank cohort, we intentionally used the previous GWAS meta-analysis, which did not include the UK Biobank data, to maintain the two-sample design [13]. Twosample MR has strength in a conservative sense over an MR analysis with overlapping samples, as the potential bias from weak instruments is toward false-negative results; thus, a positive finding from two-sample MR can be more robust evidence for the presence of causality [16]. In addition, considering the outcome sample size, applying a MR design including sample overlap was considered to cause substantial bias towards confounding effects [17] and a bias by pleiotropic effect was suspected when we used the most recent GWAS meta-analysis data as the genetic instruments for AF (Additional File 1: Supplemental Methods and Additional File 1: Supplemental Table 1).

As the outcome summary statistics for brain volume were limited to those with white British ancestry, we downloaded the summary statistics for AF in individuals of European ancestry, including 15,993 AF cases and 113,719 controls. Within the European ancestry-specific analysis, 20 SNPs in different genetic loci were reported to have a genome-wide significant $\left(P<5 \times 10^{-8}\right)$ association with the AF trait (Additional File 1: Supplemental Table 2). To ensure the independence of the instruments, we disregarded rs10800507 and rs35176054 as having weaker associations with AF but being in a linkage disequilibrium state with other variants, leaving 18 independent SNPs (within a $1 \mathrm{Mb}$ window, $r^{2}<0.001$ ). As strand alignment is uncertain for palindromic SNPs, we additionally disregarded 2 palindromic SNPs (rs2921421 and rs651386) in the main genetic instruments for AF. We also performed a sensitivity analysis preserving the two palindromic SNPs or disregarding only rs651386, which had intermediate allele frequency, as the genetic instruments for AF. We inspected the orientation of the genetic effects and secured that the direction was from exposure towards outcome variable by using Steiger filtering [18].

\section{MR assumptions}

MR analysis requires three key assumptions to be attained by the genetic instrument to demonstrate causal effects [11]. First, the relevance assumption is that the genetic instrument should be strongly associated with the exposure of interest. A previous GWAS metaanalysis already provided SNPs with strong association strength with AF. We additionally tested the association strength in the UK Biobank data in 337,138 unrelated individuals of white British ancestry who passed the sample quality control filter by calculating the explained variance for AF by polygenic score analysis [12]. The AF data were collected from the hospital admission records or main causes of death, identified by an International Classification of Diseases (ICD)-10 diagnostic code of I48 or ICD-9 diagnostic code of 4273 [12]. The explained variance for AF by polygenic score derived by the instrumented SNPs was calculated by McFadden's pseudo- $R$ square method. The $F$ statistic, which should be over 10 to avoid weak instrument bias [19], was calculated to confirm the attainment of the relevance assumption, by the below equation: $[(\mathrm{n}-\mathrm{k}-1) /(\mathrm{k})]^{*}\left[R^{2} /(1-\right.$ $\left.R^{2}\right)$ ], where $n$ represents sample size, $k$ represents number of instruments, and $R^{2}$ represents explained variance of the exposure phenotype [19]. Furthermore, we additionally validated whether the genetically predicted AF by the current instruments relevantly predicts kidney function impairment as the positive control outcome. The CKDGen GWAS meta-analysis for chronic kidney disease traits (41,395 cases and 439,303 controls, URL: https://ckdgen.imbi.uni-freiburg.de/) was assessed as the outcome data by the summary-level MR method described below [20, 21]. Chronic kidney disease was reported to be causally affected by AF in our previous MR analysis including two independent cohorts but with different genetic instruments for AF [12, 21].

Second, the independence assumption is that the genetic instrument should not be associated with confounders. To attain this assumption, we investigated whether a SNP had a strong $\left(P<1 \times 10^{-5}\right)$ association with hypertension, obesity, diabetes mellitus, dyslipidemia, and thyroid disorder in the abovementioned 337,138 white British UK Biobank participants by performing a GWAS adjusted for age, sex, agexsex, age ${ }^{2}$, and the first 10 principal components (Additional File 1: Supplemental Table 2). Furthermore, in summary-level MR, we tested the presence of directional pleiotropy by identifying MR-Egger intercepts [22]. In addition, we performed multiple pleiotropy-robust MR sensitivity analysis to derive the causal estimates with relaxation of the assumption [23]. 
Third, the exclusion restriction assumption is that the causal effect of interest should be through the studied exposure. Although a formal test for this assumption is not yet possible, the utilized median-based method can relax this assumption for up to $50 \%$ of the instrumented weights, providing sensitivity analysis for the attainment of this assumption [24].

\section{Outcome data for brain volume traits}

A recent GWAS for brain imaging traits in the UK Biobank was performed for 33,224 white British ancestry individuals aged 40 to 69 years [25]. The summary statistics for white and gray matter volumes were downloaded and used as the outcome data (URL: https:// open.win.ox.ac.uk/ukbiobank/big40/) [26]. The study identified that brain imaging phenotypes are mostly genetically trackable and reported functionally relevant genetic information associated with brain structures. The brain volume was measured by magnetic resonance imaging, and the study provided summary statistics for quantile-normalized brain volume phenotypes and for the phenotypes adjusted for head size. Among the brain volume phenotypes measured, we aimed to assess the causal estimates toward composite brain gray or white matter volume, which has been repetitively investigated in previous observational studies [6,7]. As total gray or white matter volume would generally reflect the sum of variance, this approach would secure the statistical power of an MR analysis, which is particularly important in two-sample MR, which may be biased toward falsenegative findings.

\section{Summary-level MR analysis methods}

The main MR method was the multiplicative randomeffect inverse variance-weighted method, which allows balanced pleiotropy [27].

As unbalanced pleiotropic effects may still bias the causal estimates by the inverse variance-weighted method, additional pleiotropy-robust MR sensitivity analyses are commonly performed [16, 23]. First, MR-Egger regression with bootstrapped standard error was performed with the test for the presence of directional pleiotropy or MR-Egger intercept [22]. The method has strength in that the presence of a directional pleiotropic effect can be statistically tested and that pleiotropyrobust causal estimates can be yielded. However, MREgger regression has weak statistical power, particularly when the number of instrumented SNPs is low, and can still be biased when the untestable Instrument Strength Independent of Direct Effect assumption is violated by a group of variants acting through the same pleiotropic pathway [28]. Thus, additional sensitivity analysis by the median-based method is recommended; thus, we performed the weighted median method, which allows up to $50 \%$ invalid instrumented weights [24]. Next, the MR pleiotropy residual sum and outlier test, which detects and corrects the effects of outliers, was performed when the test for global heterogeneity was significant [29]. We also performed MR-robust adjusted profile score, which provides robust causal estimates by modeling the pleiotropic effects, assuming the effects are normally distributed [30]. The summary-level MR analysis was performed by the TwoSampleMR package in $R$ (version 4.0.2, the $R$ foundation) [31].

As we tested two outcome phenotypes, gray and white matter volumes, a two-sided $P$ value $<0.05 / 2$ by the inverse variance-weighted method indicated significance in the main causality analysis for the causal estimates of $\mathrm{AF}$ on brain matter volume phenotypes. As other sensitivity analyses were performed to support the main results, we used the conventional significance threshold $(P$ $<0.05)$ to characterize a sensitivity analysis result as supportive of the main result. However, as MR-Egger regression has weak statistical power, the causal estimates by the MR-Egger method were interpreted by general effect sizes and whether a significant directional pleiotropic effect was identified by the MR-Egger intercept $P$ value $[23,32]$.

Post hoc power calculation for MR analysis was performed by an online tool (URL: https://sb452.shinyapps. io/power/), followed the method suggested by S. Burgess (URL: http://mendelianrandomization.com/) [33, 34]. We calculated beta^ ${ }^{\wedge} 2 * \mathrm{MAF}^{*}(1-\mathrm{MAF})$, where MAF indicates minor allele frequency, of each instrumented SNP and summed the values for the coefficient necessary for the power calculator. As information for prevalence ratio was unavailable, although beta should be scaled to a prevalence increase of a unit of an exposure, we used the odds ratios as the proximate of the prevalence ratio considering that the case proportion was not high (12.6\%) in the GWAS meta-analysis data which was used to generate genetic instruments. The point causal estimate towards normalized brain gray or white matter volume by the inverse variance-weighted method was used as the effect size of the causal effect.

\section{Analysis with ischemic stroke as a mediator}

Multivariable MR analysis with direct adjustment for the effects of other phenotypes of the utilized genetic instrument has been used to investigate whether the identified total effect in univariable MR is mediated by a potential phenotype [35-37]. Considering that AF is well known to be associated with ischemic stroke, we performed additional mediation analysis including the data from a GWAS meta-analysis (34,217 ischemic stroke cases 406,111 controls) from individuals of European ancestry by the MEGASTROKE consortium (URL: https://www. megastroke.org/) [38, 39]. 
To establish the causal pathways, we first tested the causal estimates of AF on ischemic stroke by the aforementioned summary-level MR methods. Finally, we calculated the direct effect of AF, predicted by the main instruments including 16 SNPs, on brain volume phenotypes by multivariable MR adjusted for the genetic effects of ischemic stroke (Additional File 1: Supplemental Table 2). Indirect effects were not calculated because linear relation between the tested variables, which is required to calculate indirect effects, was not secured as binary exposure was being tested [40]. The multivariable analysis was performed by inverse-variance-weighted method and MR-Egger regression, using the MVMR and MendelianRandomization package in $\mathrm{R}[41,42]$. Again, the Bonferronicorrected significance level $(P<0.05 / 2)$ was used to indicate significant findings in the main causal estimates, and the conventional threshold $(P<0.05)$ was used in other sensitivity analyses.

The causal estimates from ischemic stroke on brain volume phenotypes were not calculated because the available genome-wide significant 32 SNPs identified by the MEGASTROKE study explained limited variance of ischemic stroke phenotype, leading to a $F$ statistic of 9.4 indicating that a valid two-sample MR with sufficient power was impossible with the available data.

\section{Results}

\section{Genetic instrument for $\mathrm{AF}$}

None of the lead SNPs associated with AF with genomewide significance showed a strong association with a potential confounder in the UK Biobank data (Additional File 1: Supplemental Table 2). Among the 337138 individuals of white British ancestry of the UK Biobank, the median age was 58 years old, and $46.3 \%$ were males. From this group, we identified 15,446 (4.6\%) AF cases. The polygenic score calculated by the genetic instrument was strongly associated $\left(P<2 \times 10^{-16}\right)$, with phenotypical AF explaining $1.6 \%$ of the variance; thus, the $F$ statistic of the 16 SNPs in the outcome data of 33,224 individuals was 33.7. When we applied genetic instruments to the positive control outcome, kidney function impairment, genetically predicted AF was relevantly associated with a higher risk of chronic kidney disease (Additional File 1: Supplemental Table 3).

\section{Causal estimates of AF on brain volume}

The summary-level MR results indicated that genetic predisposition for AF was significantly associated with lower gray matter volume, both normalized and unnormalized (Figs. 2 and 3 and Table 1). The causal estimates were also significant by all performed MR sensitivity analysis methods, except for the MR-Egger regression

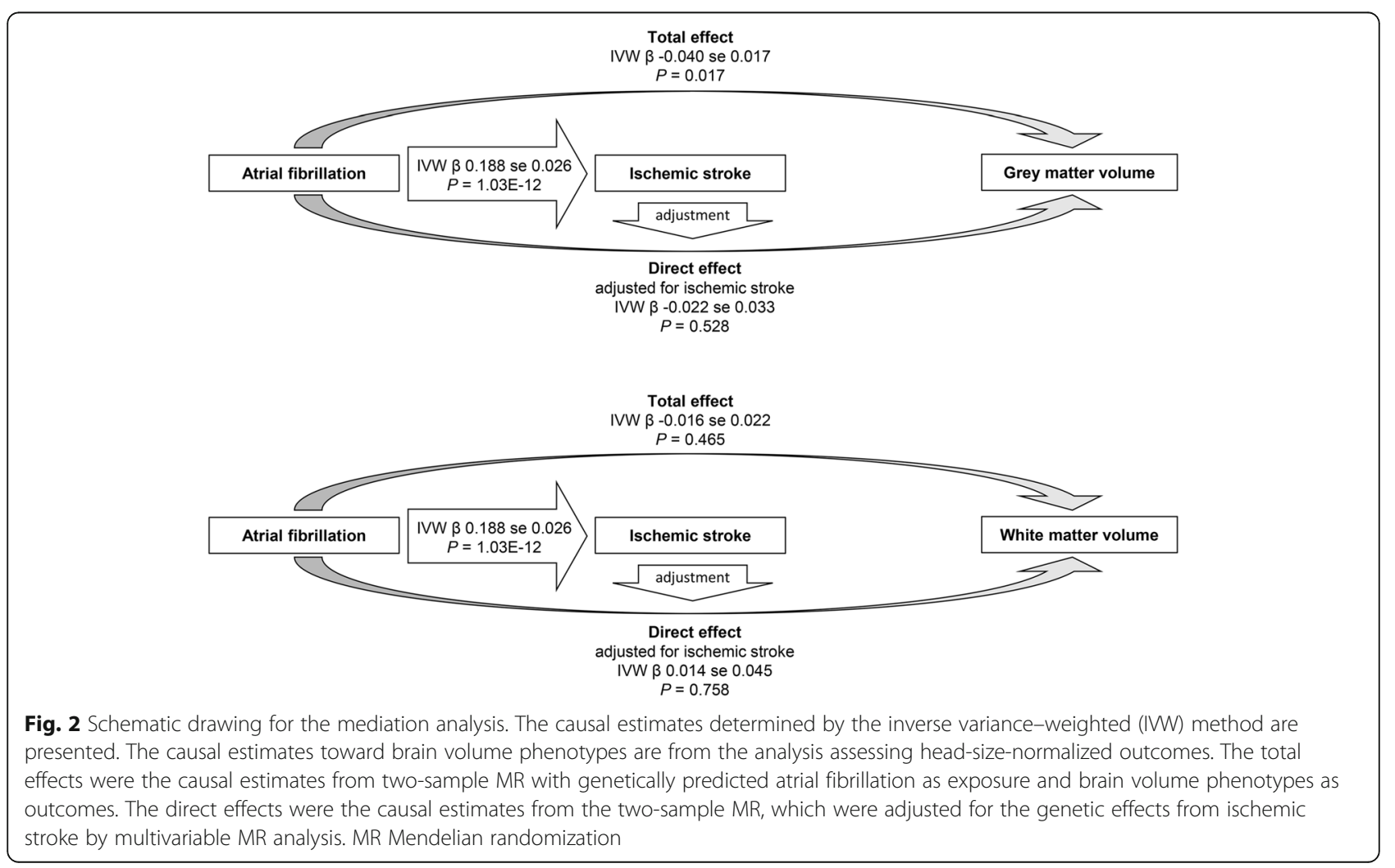




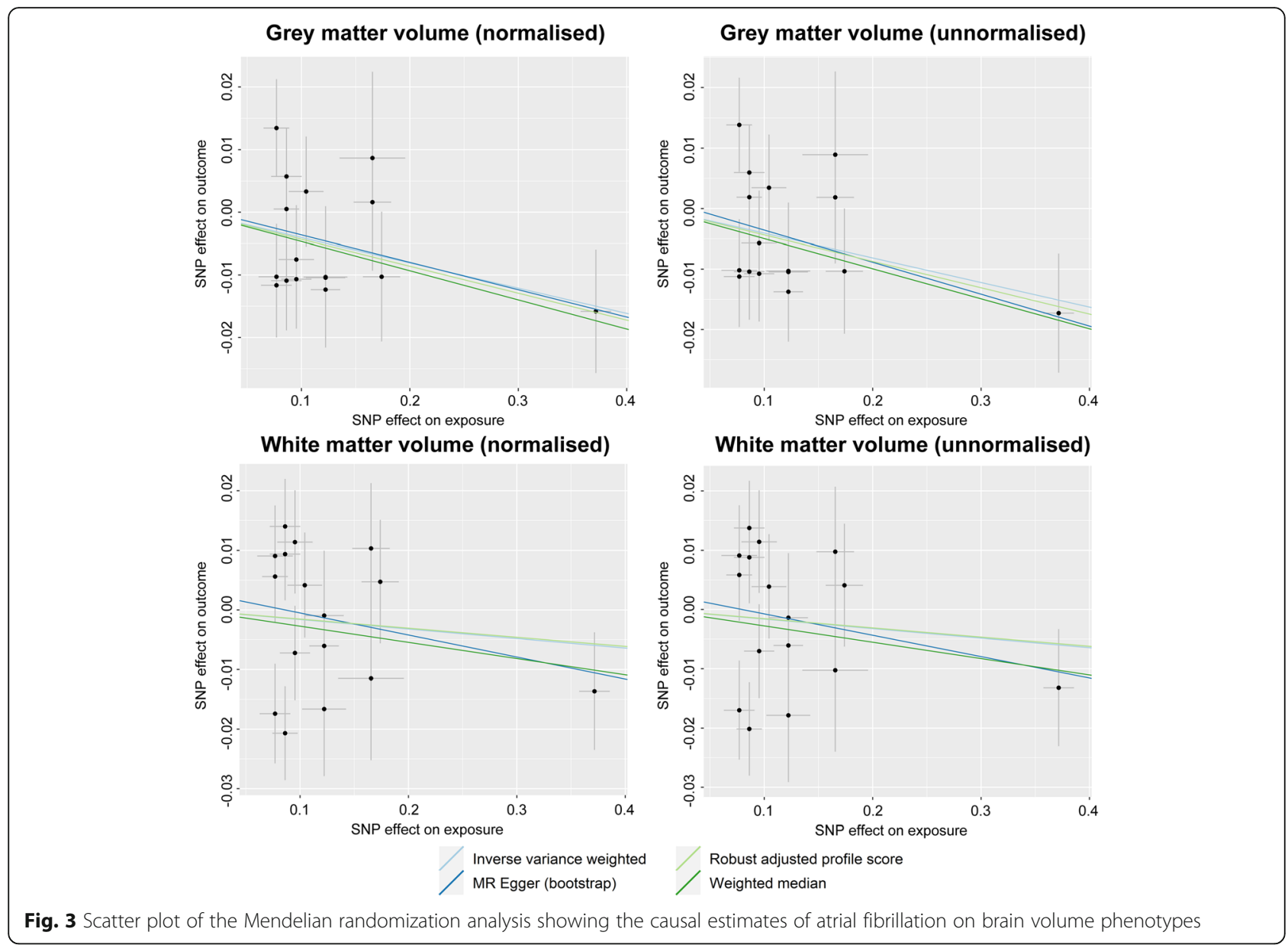

analysis. However, the MR-Egger intercept $P$ value indicated the absence of a directional pleiotropic effect, and the effect size of the causal estimates by MR-Egger regression was generally similar to the other methods. On the other hand, the causal estimates for white matter volume did not show a significant association between genetic predisposition for AF and normalized or unnormalized white matter volume by MR analysis.

When we additionally included 2 palindromic SNPs or inferred strand information to genetically predict AF, similar findings were replicated, as genetically predicted AF was significantly associated with lower brain gray matter volume (Additional File 1: Supplemental Table 4 and Additional File 1: Supplemental Table 5). The MREgger regression again yielded marginal findings with generally comparable effect sizes. Otherwise, the causal estimates toward brain white matter volume remained nonsignificant.

The power towards brain gray matter volume of the summary-level MR analysis was $79.1 \%$ when the 16 SNPs and was $87.3 \%$ when 18 SNPs, including palindromic ones, were used to genetically predict AF.
However, the power was weak towards brain white matter volume, as it was $23.6 \%$ by 18 SNPs and $19.7 \%$ by 16 SNPs, respectively.

\section{$\mathrm{AF}$, ischemic stroke, and brain volume}

The causal estimates of AF on ischemic stroke indicated a significant causal effect, and the causal estimates were significant by all performed MR analysis methods (Table 2 and Fig. 2). Although a directional pleiotropic effect was suspected by the MR-Egger intercept $P$ value (directional pleiotropy $P=0.028$ ), MR-Egger regression correcting the potential pleiotropic effect still provided significant causal estimates.

Finally, when the abovementioned total effects from $\mathrm{AF}$ on brain volume phenotypes were adjusted for the genetic effects of ischemic stroke by multivariable MR, the causal estimates on brain gray matter volume were attenuated to the point of nonsignificance (Table 3 and Fig. 2). Namely, the direct effects of AF on brain gray matter volume were nonsignificant independent of the effects of ischemic stroke. The causal estimates by the multivariable MR analysis for white matter volume 
Table 1 Causal estimates of atrial fibrillation on brain gray or white matter volume in the UK Biobank participants by summary-level Mendelian randomization

\begin{tabular}{|c|c|c|c|c|c|c|c|}
\hline $\begin{array}{l}\text { Genetically predicted } \\
\text { exposure }\end{array}$ & Outcome phenotype & $\begin{array}{l}\text { MR-Egger } \\
\text { intercept } P \text { value }\end{array}$ & $\begin{array}{l}\text { Cochran's } Q \text { statistic } \\
P \text { value for heterogeneity }\end{array}$ & MR method & Beta & $\begin{array}{l}\text { Standard } \\
\text { error }\end{array}$ & $\begin{array}{l}P \\
\text { value }\end{array}$ \\
\hline \multirow{20}{*}{$\begin{array}{l}\text { Atrial fibrillation (16 } \\
\text { SNPs) }\end{array}$} & \multirow{5}{*}{$\begin{array}{l}\text { Gray matter volume } \\
\text { (normalized) }\end{array}$} & \multirow[t]{5}{*}{0.984} & \multirow[t]{5}{*}{0.547} & IVW & -0.040 & 0.017 & 0.017 \\
\hline & & & & $\begin{array}{l}\text { MR-Egger } \\
\text { (bootstrap) }\end{array}$ & -0.044 & 0.040 & 0.147 \\
\hline & & & & $\begin{array}{l}\text { Weighted } \\
\text { median }\end{array}$ & -0.047 & 0.023 & 0.044 \\
\hline & & & & MR-RAPS & -0.043 & 0.017 & 0.014 \\
\hline & & & & MR-PRESSO & NA & NA & NA \\
\hline & \multirow{5}{*}{$\begin{array}{l}\text { Gray matter volume } \\
\text { (unnormalized) }\end{array}$} & \multirow[t]{5}{*}{0.817} & \multirow[t]{5}{*}{0.499} & IVW & -0.041 & 0.017 & 0.015 \\
\hline & & & & $\begin{array}{l}\text { MR-Egger } \\
\text { (bootstrap) }\end{array}$ & -0.052 & 0.040 & 0.089 \\
\hline & & & & $\begin{array}{l}\text { Weighted } \\
\text { median }\end{array}$ & -0.050 & 0.023 & 0.033 \\
\hline & & & & MR-RAPS & -0.044 & 0.017 & 0.013 \\
\hline & & & & MR-PRESSO & NA & NA & NA \\
\hline & \multirow{5}{*}{$\begin{array}{l}\text { White matter volume } \\
\text { (normalized) }\end{array}$} & \multirow[t]{5}{*}{0.549} & \multirow[t]{5}{*}{0.041} & IVW & -0.016 & 0.022 & 0.465 \\
\hline & & & & $\begin{array}{l}\text { MR-Egger } \\
\text { (bootstrap) }\end{array}$ & -0.038 & 0.042 & 0.186 \\
\hline & & & & $\begin{array}{l}\text { Weighted } \\
\text { median }\end{array}$ & -0.027 & 0.023 & 0.242 \\
\hline & & & & MR-RAPS & -0.015 & 0.022 & 0.483 \\
\hline & & & & MR-PRESSO & NA & NA & NA \\
\hline & \multirow{5}{*}{$\begin{array}{l}\text { White matter volume } \\
\text { (unnormalized) }\end{array}$} & \multirow[t]{5}{*}{0.553} & \multirow[t]{5}{*}{0.053} & IWW & -0.016 & 0.022 & 0.464 \\
\hline & & & & $\begin{array}{l}\text { MR-Egger } \\
\text { (bootstrap) }\end{array}$ & -0.038 & 0.041 & 0.181 \\
\hline & & & & $\begin{array}{l}\text { Weighted } \\
\text { median }\end{array}$ & -0.028 & 0.024 & 0.241 \\
\hline & & & & MR-RAPS & -0.016 & 0.022 & 0.470 \\
\hline & & & & MR-PRESSO & NA & NA & NA \\
\hline
\end{tabular}

MR Mendelian randomization, IVW inverse variance-weighted, RAPS robust adjusted profile score, PRESSO pleiotropy residual sum and outlier, SNP single-nucleotide polymorphism

MR-PRESSO analysis was performed, but the MR-PRESSO global test for heterogeneity did not identify correctable effects of outliers

The units of the causal estimates were log odds ratio for the cause (atrial fibrillation) and standard deviation for the effect (brain volume)

Table 2 Causal estimates of atrial fibrillation on ischemic stroke

\begin{tabular}{|c|c|c|c|c|c|c|c|}
\hline $\begin{array}{l}\text { Genetically predicted } \\
\text { exposure }\end{array}$ & $\begin{array}{l}\text { Outcome } \\
\text { phenotype }\end{array}$ & $\begin{array}{l}\text { MR-Egger intercept } \\
P \text { value }\end{array}$ & $\begin{array}{l}\text { Cochran's } Q \text { statistic } \\
P \text { value for heterogeneity }\end{array}$ & MR method & Beta & $\begin{array}{l}\text { Standard } \\
\text { error }\end{array}$ & $\begin{array}{l}P \\
\text { value }\end{array}$ \\
\hline \multirow[t]{5}{*}{ Atrial fibrillation (16 SNPs) } & Ischemic stroke & 0.028 & 0.068 & IVW & 0.188 & 0.026 & $\begin{array}{l}1.03 \mathrm{E} \\
-12\end{array}$ \\
\hline & & & & $\begin{array}{l}\text { MR-Egger } \\
\text { (bootstrap) }\end{array}$ & 0.251 & 0.047 & $\begin{array}{l}< \\
0.001\end{array}$ \\
\hline & & & & $\begin{array}{l}\text { Weighted } \\
\text { median }\end{array}$ & 0.240 & 0.030 & $\begin{array}{l}4.83 \mathrm{E} \\
-16\end{array}$ \\
\hline & & & & MR-RAPS & 0.201 & 0.025 & $\begin{array}{l}5.57 \mathrm{E} \\
-16\end{array}$ \\
\hline & & & & MR-PRESSO & NA & NA & NA \\
\hline
\end{tabular}

MR Mendelian randomization, IVW inverse variance-weighted, RAPS robust adjusted profile score, PRESSO pleiotropy residual sum and outlier, SNP single-nucleotide polymorphism

MR-PRESSO analysis was performed, but as the MR-PRESSO global test for heterogeneity did not identify correctable effects of outliers, the causal estimates were the same as those of the inverse variance-weighted method

The units of the causal estimates were log odds ratio for the cause (atrial fibrillation) and log odds ratio for the effect (stroke) 
Table 3 Direct effects of atrial fibrillation on brain volume phenotypes adjusted for genetic effects of ischemic stroke by multivariable MR analysis.

\begin{tabular}{|c|c|c|c|c|c|c|c|c|}
\hline Genetically predicted exposure & $\begin{array}{l}\text { Conditional } \\
F \text { statistics } \\
\text { (atrial fibrillation) }\end{array}$ & $\begin{array}{l}\text { Conditional } \\
\text { F statistics } \\
\text { (stroke) } \\
\end{array}$ & $\begin{array}{l}\text { Outcome } \\
\text { phenotype }\end{array}$ & $\begin{array}{l}\text { Heterogeneity } \\
Q \text { statistics } \\
P \text { value }\end{array}$ & Method & Beta & $\begin{array}{l}\text { Standard } \\
\text { error }\end{array}$ & $\begin{array}{l}P \\
\text { value }\end{array}$ \\
\hline \multirow{8}{*}{$\begin{array}{l}\text { Atrial fibrillation, adjusted for genetic } \\
\text { effects of ischemic stroke ( } 16 \text { SNPs) }\end{array}$} & \multirow[t]{8}{*}{1.734} & \multirow[t]{8}{*}{1.406} & \multirow{2}{*}{$\begin{array}{l}\text { Gray matter } \\
\text { volume } \\
\text { (normalized) }\end{array}$} & \multirow[t]{2}{*}{0.443} & IVW & -0.022 & 0.033 & 0.528 \\
\hline & & & & & $\begin{array}{l}\text { MR- } \\
\text { Egger }\end{array}$ & -0.001 & 0.065 & 0.992 \\
\hline & & & \multirow{2}{*}{$\begin{array}{l}\text { Gray matter } \\
\text { volume } \\
\text { (unnormalized) }\end{array}$} & \multirow[t]{2}{*}{0.397} & IVW & -0.022 & 0.034 & 0.533 \\
\hline & & & & & $\begin{array}{l}\text { MR- } \\
\text { Egger }\end{array}$ & -0.015 & 0.066 & 0.823 \\
\hline & & & \multirow{2}{*}{$\begin{array}{l}\text { White matter } \\
\text { volume } \\
\text { (normalized) }\end{array}$} & \multirow[t]{2}{*}{0.035} & IVW & 0.014 & 0.045 & 0.758 \\
\hline & & & & & $\begin{array}{l}\text { MR- } \\
\text { Egger }\end{array}$ & -0.003 & 0.088 & 0.971 \\
\hline & & & \multirow{2}{*}{$\begin{array}{l}\text { White matter } \\
\text { volume } \\
\text { (unnormalized) }\end{array}$} & \multirow[t]{2}{*}{0.043} & IVW & 0.013 & 0.045 & 0.777 \\
\hline & & & & & $\begin{array}{l}\text { MR- } \\
\text { Egger }\end{array}$ & -0.005 & 0.087 & 0.958 \\
\hline
\end{tabular}

MR Mendelian randomization, IVW inverse variance-weighted, SNP single-nucleotide polymorphism

Multivariable inverse variance-weighted method and MR-Egger regression was performed to yield the causal estimates

remained nonsignificant as the total effects. However, the overall conditioned $F$ statistics indicated the possibility of weak instruments in the multivariable MR anlaysis, suggesting that it was difficult to independently predict the atrial fibrillation and ischemic stroke phenotypes.

\section{Discussion}

This study identified that genetic predisposition for AF is significantly associated with lower gray matter volume but not white matter volume. With our efforts to attain the MR assumptions, our study supports that AF is a causative factor for lower gray matter volume. The results further suggest causal pathways from AF to low gray matter volume may be mediated by ischemic stroke.

An observational association between AF and functional brain disorders, representatively dementia, has been reported [5, 9]. Historically, loss in brain gray matter volume and increase in abnormal white matter volume were considered to be associated with dementia severity [43]. As AF was associated with lower gray matter volume by observational findings $[6,7]$, structural brain atrophy caused by AF was suspected to be one of the mechanisms of cognitive impairment in AF patients. In addition, as AF is a widely recognized risk factor for ischemic stroke, either by cardioembolism or not [44], and ischemic stroke has been reported to be associated with accelerated brain atrophy [45], conceptional linkage between AF, ischemic stroke, and brain volume loss has been suggested. On the other hand, there have been debates regarding whether AF may be associated with brain volume loss or the risk of dementia independent of stroke-related mechanisms [7, 9]. However, confirming the causal linkage between AF, ischemic stroke, and brain volume loss was difficult by traditional study designs, as observational findings are prone to residual confounding effects or reverse causation [11]. Moreover, as brain volume is a relatively unique phenotype available in a large number of individuals and rarely sequentially measured, an observational study investigating the causal effect of AF on brain volume is difficult to perform. In this study, we implemented MR analysis to test causal estimates from exposure and predicted complex outcomes using the genetic instrument. Finally, we identified that AF may be causally linked to a lower gray matter volume. The identified causal estimates were dependent on the effect of ischemic stroke; thus, the study suggests causal pathways linking AF, ischemic stroke, and lower brain gray matter volume.

Based on the study results, as AF is a causative factor for lower brain gray matter volume, appropriate AF management might delay dementia-related brain atrophy. Considering that the identified causal pathway may be mediated by ischemic stroke, conventional clinical interventions lowering the risk of ischemic stroke in AF patients may be beneficial for preventing accelerated loss of gray matter volume. Recent studies reported that AF patients who received a rhythm-control strategy exhibited a lower risk of cognitive function impairment, supporting that the identified causal pathway may be extended toward cognitive function outcome [46, 47]. A future study would be needed to test our hypothesis that appropriate anticoagulative therapy or rhythm-control intervention in patients with AF would decrease the risk of brain volume loss in patients with AF. In addition, considering that subclinical AF and early brain disorders are often underdiagnosed, AF screening in the high-risk elderly population with brain atrophy or 
cognitive dysfunction would be considered to facilitate early diagnosis of the suggested causal factor.

In this study, the multivariable MR analysis indicated that it was difficult to independently predict the two closely tight phenotypes, AF and ischemic stroke, by genetic information considering the low conditioned $F$ statistics. However, if we allow the interpretation for the results considering the effects from AF on brain volume is hardly explained apart from the close bound between AF and ischemic stroke, suggests that the strokemediated pathway would be the prioritized biological linkage rather than a stroke-independent effect of AF on low brain gray matter volume. Previous observational findings suggest that AF was associated with dementia or brain volume independent of stroke might have been potentially affected by confounding effects, as AF commonly occurs in individuals with multiple underlying diseases $[8,9]$. However, caution is necessary for such interpretation, as the modest degree of direct effect of AF on brain volume phenotypes, not mediated by ischemic stroke, could have been unrevealed because of the potential false-negative bias in two-sample MR [16], particularly considering the low conditioned $F$ statistics of AF phenotype when adjusted for the genetic effects towards ischemic stroke trait. As AF is linked to diverse biological consequences, including subclinical brain hemodynamic compromises or neurohormonal responses $[8,48,49]$, a future study is still necessary to investigate whether ischemic stroke-independent effects of AF on brain volume or cognitive function are present.

There are several limitations to this study. First, the study assumes that the brain volumes of middle to elderly UK Biobank participants were determined by degenerative processes later in life. Although this assumption may be attained because brain volume changes are largely affected by risk factors for degenerative diseases [50], it is still possible that developmental, rather than degenerative, determinants might have affected the brain volume phenotypes. To overcome this issue, we also investigated the brain volume phenotypes adjusted for head size, which may partially reflect some developmental differences. However, it should be noted that attainment of the exclusion-restriction assumption, which is untestable, is required to consider our MR analysis results valid. Second, we assessed binary AF exposure in this MR analysis; however, AF has diverse severity and status. Thus, whether the effects of AF are different according to the subtypes (e.g., paroxysmal or persistent) could not be studied herein. Third, the study is mainly based on data from individuals of European ancestry; thus, generalizability to other ethnic populations is not guaranteed. MR results can also be affected by selection bias; thus, future replication studies may be necessary to confirm that the findings could be applied to the general population. Fourth, as the effect size of the causal estimates from AF towards brain white matter volume was modest than that towards brain gray matter volume, statistical power was weak for the phenotype. As twosample MR can be biased towards false-negative finding, a modest degree of causal effect from AF towards brain white matter volume cannot be disregarded from this study. Lastly, the multivariable MR analysis used instruments with low conditioned $F$ statistics. Also, the causal estimates from stroke phenotypes on brain volume were not calculated because of the limited availability of instruments. This limitation of the available dataset should be overcome in future studies to clearly dissect the linkage between $\mathrm{AF}$, ischemic stroke, and brain volumes.

\section{Conclusions}

In conclusion, our MR results suggested that AF was causally linked to a lower brain gray matter volume, possibly mediated by the effects of ischemic stroke. A future study is warranted to investigate whether appropriate AF management may result in delayed progression of gray matter volume loss in AF patients.

\section{Abbreviations}

AF: Atrial fibrillation; GWAS: Genome-wide association study; ICD: International classification of diseases; MAF: Minor allele frequency; MR: Mendelian randomization; SNP: Single-nucleotide polymorphism

\section{Supplementary Information}

The online version contains supplementary material available at https://doi. org/10.1186/s12916-021-02152-9.

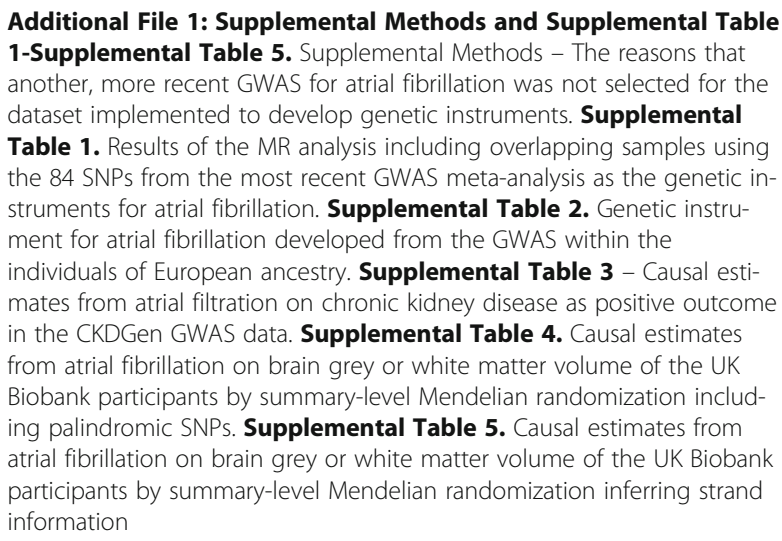

\section{Acknowledgements \\ The study was based on the data provided by the UK Biobank consortium (application No. 53799), the Cardiovascular Disease Knowledge Portal, and the MEGASTROKE project. The MEGASTROKE project received funding from sources specified at http://www.megastroke.org/acknowledgments.html. We thank the investigators of the previous studies who provided the valuable genetic summary statistics for this study.}

Data access, responsibility, and analysis

Dr. Dong Ki Kim and Dr. Sehoon Park had full access to all data in the study and take responsibility for the integrity of the data and the accuracy of the data analysis. 


\section{Authors' contributions}

The corresponding author attests that all listed authors meet the authorship criteria and that no others meeting the criteria have been omitted. SP, HL, KK, KWJ, and DKK contributed to the conception and design of the study. SL, YK, SC, YCK, SL, EKC, SSH, JPL, SL, EKC, KWJ, CSL, YSK, and DKK provided statistical advice and interpreted the data. SP and KK performed the main statistical analysis, assisted by SL, and YK. HL, JPL, KWJ, CSL, YSK, and DKK provided advice regarding the data interpretation. YCK, SSH, HL, JPL, KWJ, CSL, and YSK provided material support during the study. All authors participated in drafting the manuscript. The authors reviewed the manuscript and approved the final version to be published.

\section{Funding}

This work was supported by a grant from the Korea Health Technology R\&D Project through the Korea Health Industry Development Institute (KHIDI), funded by the Ministry of Health \& Welfare, Republic of Korea (grant number: HW20C2066).

The funder played no role in the conduct of the study, and the study was performed independently by the authors.

\section{Availability of data and materials}

The data for this study are available in the public domain, as described in the manuscript.

\section{Declarations}

\section{Ethics approval and consent to participate}

The study was approved by the institutional review boards of the Seoul National University Hospital (No. E-2012-004-1177) and the UK Biobank consortium (application No. 53799). The study was performed in accordance with the Declaration of Helsinki. The requirement for informed consent was waived because the study analyzed public databases.

\section{Consent for publication}

Not applicable

\section{Competing interests}

EKC: research grants from Bayer, BMS/Pfizer, Biosense Webster, Chong Kun Dang, Daiichi-Sankyo, Dreamtech Co., Ltd., Medtronic, Samjinpharm, SanofiAventis, Seers Technology, Skylabs, and Yuhan. No fees are received personally. The other authors declare no competing interests.

\section{Author details}

'Department of Biomedical Sciences, Seoul National University College of Medicine, Seoul, Korea. ${ }^{2}$ Department of Internal Medicine, Armed Forces Capital Hospital, Gyeonggi-do, Seongnam, Korea. ${ }^{3}$ Department of Internal Medicine, Department of Internal Medicine, Uijeongbu Eulji University Medical Center, Gyeonggi-do, Uijeongbu, Korea. ${ }^{4}$ Department of Internal Medicine, Seoul National University Hospital, 101 Daehak-ro, Jongno-gu, Seoul 03080, Korea. ${ }^{5}$ Department of Internal Medicine, Seoul National University College of Medicine, Seoul, Korea. ${ }^{6}$ Department of Internal Medicine, Keimyung University School of Medicine, Daegu, Korea. ${ }^{7}$ Transdisciplinary Department of Medicine \& Advanced Technology, Seoul National University Hospital, Seoul, Korea. ${ }^{8}$ Kidney Research Institute, Seoul National University, Seoul, Korea. ${ }^{9}$ Department of Internal Medicine, Seoul National University Boramae Medical Center, Seoul, Korea.

\section{Received: 22 March 2021 Accepted: 4 October 2021}

Published online: 24 November 2021

\section{References}

1. Chugh SS, Havmoeller R, Narayanan K, Singh D, Rienstra M, Benjamin EJ, Gillum RF, Kim YH, McAnulty JH, Jr., Zheng ZJ et al: Worldwide epidemiology of atrial fibrillation: a Global Burden of Disease 2010 Study. Circulation 2014, 129(8):837-847 https://doi.org/https://doi.org/10.1161/ circulationaha.113.005119

2. Wang L, Ze F, Li J, Mi L, Han B, Niu H, Zhao N: Trends of global burden of atrial fibrillation/flutter from Global Burden of Disease Study 2017. Heart 2021, 107(11):881-887 https://doi.org/https://doi.org/10.1136/heartjnl-202 0-317656
3. Rahman F, Kwan GF, Benjamin EJ: Global epidemiology of atrial fibrillation. Nat Rev Cardiol 2016, 13(8):501 https://doi.org/https://doi.org/10.1038/nrca rdio.2016.114

4. Lee SR, Choi EK, Han KD, Cha MJ, Oh S: Trends in the incidence and prevalence of atrial fibrillation and estimated thromboembolic risk using the CHA(2)DS(2)-VASC score in the entire Korean population. Int J Cardiol 2017, 236:226-231 https://doi.org/https://doi.org/10.1016/j.ijcard.2017.02.039

5. Kalantarian S, Stern TA, Mansour M, Ruskin JN: Cognitive impairment associated with atrial fibrillation: a meta-analysis. Ann Intern Med 2013, 158(5 Pt 1):338-346 https://doi.oerg/https://doi.org/10.7326/0003-4819-1585-201303050-00007

6. Moazzami K, Shao IY, Chen LY, Lutsey PL, Jack CR, Jr., Mosley T, Joyner DA, Gottesman R, Alonso A: Atrial fibrillation, brain volumes, and subclinical cerebrovascular disease (from the atherosclerosis risk in communities neurocognitive study [ARIC-NCS]). Am J Cardiol 2020, 125(2):222-228 https:/ doi.org/https://doi.org/10.1016/j.amjcard.2019.10.010

7. Stefansdottir H, Arnar DO, Aspelund T, Sigurdsson S, Jonsdottir MK, Hjaltason H, Launer L, Gudnason V: Atrial fibrillation is associated with reduced brain volume and cognitive function independent of cerebral infarcts. Stroke 2013, 44(4):1020-1025 https://doi.org/https://doi.org/10.1161/ strokeaha.12.679381

8. Gardarsdottir M, Sigurdsson S, Aspelund T, Rokita H, Launer L, Gudnason V, Arnar DO: Atrial fibrillation is associated with decreased total cerebral blood flow and brain perfusion. Europace 2018, 20(8):1252-1258 https://doi.org/ https://doi.org/10.1093/europace/eux220

9. Kim D, Yang PS, Yu HT, Kim TH, Jang E, Sung JH, Pak HN, Lee MY, Lee MH, Lip GYH, Joung B: Risk of dementia in stroke-free patients diagnosed with atrial fibrillation: data from a population-based cohort. Eur Heart J 2019, 40(28):2313-2323 https://doi.org/https://doi.org/10.1093/eurheartj/ehz386

10. Werden E, Cumming T, Li Q, Bird L, Veldsman M, Pardoe HR, Jackson $G$, Donnan GA, Brodtmann A: Structural MRI markers of brain aging early after ischemic stroke. Neurology 2017, 89(2):116-124 https://doi.org/https://doi. org/10.1212/wnl.0000000000004086

11. Davies NM, Holmes MV, Davey Smith G: Reading Mendelian randomisation studies: a guide, glossary, and checklist for clinicians. BMJ 2018, 362:k601 https://doi.org/https://doi.org/10.1136/bmj.k601

12. Park S, Lee S, Kim Y, Lee Y, Kang MW, Kim K, Kim YC, Han SS, Lee H, Lee JP, Joo KW, Lim CS, Kim YS, Kim DK: Atrial fibrillation and kidney function: a bidirectional Mendelian randomization study. Eur Heart J 2021, 42(29):28162823 https://doi.org/https://doi.org/10.1093/eurheartj/ehab291

13. Christophersen IE, Rienstra M, Roselli C, Yin X, Geelhoed B, Barnard J, Lin H, Arking DE, Smith AV, Albert CM et al: Large-scale analyses of common and rare variants identify 12 new loci associated with atrial fibrillation. Nat Genet 2017, 49(6):946-952 https://doi.org/https://doi.org/10.1038/ng.3843

14. Christophersen IE, Rienstra M, Roselli C, Yin X, Geelhoed B, Barnard J, Lin H, Arking DE, Smith AV, Albert CM et al. Cardiovascular Disease Knowledge Portal. https://cvd.hugeamp.org/ (2017)

15. Roselli C, Chaffin MD, Weng LC, Aeschbacher S, Ahlberg G, Albert CM, Almgren P, Alonso A, Anderson CD, Aragam KG, Arking DE, Barnard J, Bartz TM, Benjamin EJ, Bihlmeyer NA, Bis JC, Bloom HL, Boerwinkle E, Bottinger EB, Brody JA, Calkins H, Campbell A, Cappola TP, Carlquist J, Chasman DI, Chen LY, Chen YDI, Choi EK, Choi SH, Christophersen IE, Chung MK, Cole JW, Conen D, Cook J, Crijns HJ, Cutler MJ, Damrauer SM, Daniels BR, Darbar D, Delgado G, Denny JC, Dichgans M, Dörr M, Dudink EA, Dudley SC, Esa N, Esko T, Eskola M, Fatkin D, Felix SB, Ford I, Franco OH, Geelhoed B, Grewal RP, Gudnason V, Guo X, Gupta N, Gustafsson S, Gutmann R, Hamsten A, Harris TB, Hayward C, Heckbert SR, Hernesniemi J, Hocking L, Hofman A, Horimoto ARVR, Huang J, Huang PL, Huffman J, Ingelsson E, Ipek EG, Ito K, Jimenez-Conde J, Johnson R, Jukema JW, Kääb S, Kähönen M, Kamatani Y, Kane JP, Kastrati A, Kathiresan S, Katschnig-Winter P, Kavousi M, Kessler T, Kietselaer BL, Kirchhof P, Kleber ME, Knight S, Krieger JE, Kubo M, Launer LJ, Laurikka J, Lehtimäki T, Leineweber K, Lemaitre RN, Li M, Lim HE, Lin HJ, Lin H, Lind L, Lindgren CM, Lokki ML, London B, Loos RJF, Low SK, Lu Y, Lyytikäinen LP, Macfarlane PW, Magnusson PK, Mahajan A, Malik R, Mansur AJ, Marcus GM, Margolin L, Margulies KB, März W, McManus DD, Melander O, Mohanty S, Montgomery JA, Morley MP, Morris AP, Müller-Nurasyid M, Natale A, Nazarian S, Neumann B, Newton-Cheh C, Niemeijer MN, Nikus K, Nilsson P, Noordam R, Oellers H, Olesen MS, Orho-Melander M, Padmanabhan S, Pak HN, Paré G, Pedersen NL, Pera J, Pereira A, Porteous D, Psaty BM, Pulit SL, Pullinger CR, Rader DJ, Refsgaard L, Ribasés M, Ridker PM, Rienstra M, Risch L, Roden DM, Rosand J, Rosenberg MA, Rost N, Rotter Jl, 
Saba S, Sandhu RK, Schnabel RB, Schramm K, Schunkert H, Schurman C, Scott SA, Seppälä I, Shaffer C, Shah S, Shalaby AA, Shim J, Shoemaker MB, Siland JE, Sinisalo J, Sinner MF, Slowik A, Smith AV, Smith BH, Smith JG, Smith JD, Smith NL, Soliman EZ, Sotoodehnia N, Stricker BH, Sun A, Sun H, Svendsen JH, Tanaka T, Tanriverdi K, Taylor KD, Teder-Laving M, Teumer A, Thériault S, Trompet S, Tucker NR, Tveit A, Uitterlinden AG, van der Harst $\mathrm{P}$, van Gelder IC, van Wagoner DR, Verweij N, Vlachopoulou E, Völker U, Wang B, Weeke PE, Weijs B, Weiss R, Weiss S, Wells QS, Wiggins KL, Wong JA, Woo D, Worrall BB, Yang PS, Yao J, Yoneda ZT, Zeller T, Zeng L, Lubitz SA, Lunetta KL, Ellinor PT: Multi-ethnic genome-wide association study for atrial fibrillation. Nat Genet 2018, 50(9):1225-1233 https://doi.org/https://doi.org/1 0.1038/s41588-018-0133-9

16. Burgess S, Davey Smith G, Davies NM, Dudbridge F, Gill D, Glymour MM, Hartwig FP, Holmes MV, Minelli C, Relton CL, Theodoratou E: Guidelines for performing Mendelian randomization investigations. Wellcome Open Res 2019, 4:186 https://doi.org/https://doi.org/10.12688/wellcomeopenres.1 5555.2

17. Minelli C, Del Greco MF, van der Plaat DA, Bowden J, Sheehan NA, Thompson J: The use of two-sample methods for Mendelian randomization analyses on single large datasets. Int J Epidemiol 2021 [Epub ahead of print] https://doi.org/https://doi.org/10.1093/ije/dyab084

18. Hemani G, Tilling K, Davey Smith G: Orienting the causal relationship between imprecisely measured traits using GWAS summary data. PLOS Genet 2017, 13(11):e1007081 https://doi.org/https://doi.org/10.1371/journal. pgen.1007081

19. Burgess S, Thompson SG: Avoiding bias from weak instruments in Mendelian randomization studies. Int J Epidemiol 2011, 40(3):755-764 https://doi.org/https://doi.org/10.1093/ije/dyr036

20. Wuttke M, Li Y, Li M, Sieber KB, Feitosa MF, Gorski M, Tin A, Wang L, Chu AY, Hoppmann A, et al. CKDGen Consortium - Meta-analysis Data. https:// ckdgen.imbi.uni-freiburg.de/.

21. Wuttke M, Li Y, Li M, Sieber KB, Feitosa MF, Gorski M, Tin A, Wang L, Chu AY, Hoppmann A et al: A catalog of genetic loci associated with kidney function from analyses of a million individuals. Nat Genet 2019, 51(6):957972 https://doi.org/https://doi.org/10.1038/s41588-019-0407-x

22. Bowden J, Davey Smith G, Burgess S: Mendelian randomization with invalid instruments: effect estimation and bias detection through Egger regression. Int J Epidemiol 2015, 44(2):512-525 https://doi.org/https://doi.org/10.1093/ ije/dyv080

23. Slob EAW, Burgess S: A comparison of robust Mendelian randomization methods using summary data. Genet Epidemiol 2020, 44(4):313-329 https:// doi.org/https://doi.org/https://doi.org/10.1002/gepi.22295

24. Bowden J, Davey Smith G, Haycock PC, Burgess S: Consistent estimation in Mendelian randomization with some invalid instruments using a weighted median estimator. Genet Epidemiol 2016, 40(4):304-314 https://doi.org/ https://doi.org/10.1002/gepi.21965

25. Smith SM, Douaud G, Chen W, Hanayik T, Alfaro-Almagro F, Sharp K, Elliott LT: An expanded set of genome-wide association studies of brain imaging phenotypes in UK Biobank. Nat Neurosci 2021, 24(5):737-745 https:/doi.org/ https://doi.org/10.1038/s41593-021-00826-4

26. Stephen M. Smith GD, Winfield Chen, Taylor Hanayik, Fidel Alfaro-Almagro, Kevin Sharp, Lloyd T. Elliott. Oxford Brain Imaging Genetics Server - BIG40. https://open.win.ox.ac.uk/ukbiobank/big40/ (2021)

27. Zhao JV, Schooling CM: Effect of linoleic acid on ischemic heart disease and its risk factors: a Mendelian randomization study. BMC Med 2019, 17(1):61 https://doi.org/https://doi.org/10.1186/s12916-019-1293-x

28. Burgess $\mathrm{S}$, Thompson SG: Interpreting findings from Mendelian randomization using the MR-Egger method. Eur J Epidemiol 2017, 32(5): 377-389 https://doi.org/https://doi.org/10.1007/s10654-017-0255-x

29. Verbanck M, Chen CY, Neale B, Do R: Detection of widespread horizontal pleiotropy in causal relationships inferred from Mendelian randomization between complex traits and diseases. Nat Genet 2018, 50(5):693-698 https:// doi.org/https://doi.org/10.1038/541588-018-0099-7

30. Zhao Q, Wang J, Hemani G, Bowden J, Small DS. Statistical inference in twosample summary-data Mendelian randomization using robust adjusted profile score. Ann Stat. 2020;48(3):1742-1769, 1728 https://doi.org/. https:// doi.org/10.1214/19-AOS1866.

31. Hemani $\mathrm{G}$, Zheng J, Elsworth B, Wade KH, Haberland V, Baird D, Laurin C, Burgess S, Bowden J, Langdon R, Tan VY, Yarmolinsky J, Shihab HA, Timpson NJ, Evans DM, Relton C, Martin RM, Davey Smith G, Gaunt TR, Haycock PC: The MR-Base platform supports systematic causal inference across the human phenome. Elife 2018, 7 https://doi.org/https://doi.org/10. 7554/eLife.34408

32. Park S, Lee S, Kim Y, Lee Y, Kang MW, Kim K, Kim YC, Han SS, Lee H, Lee JP, Joo KW, Lim CS, Kim YS, Kim DK: Causal effects of positive affect, life satisfaction, depressive symptoms, and neuroticism on kidney function: a Mendelian randomization study. J Am Soc Nephrol 2021, 32(6):1484-1496 https://doi.org/https://doi.org/10.1681/asn.2020071086

33. Burgess $\mathrm{S}$, Labrecque JA: Mendelian randomization with a binary exposure variable: interpretation and presentation of causal estimates. Eur J Epidemio 2018, 33(10):947-952 https://doi.org/https://doi.org/10.1007/s10654-0180424-6

34. Zeng H, Ge J, Xu W, Ma H, Chen L, Xia M, Pan B, Lin H, Wang S, Gao X: Type 2 diabetes is causally associated with reduced serum osteocalcin: a genomewide association and Mendelian randomization study. J Bone Miner Res 2021, 36(9):1694-1707 https://doi.org/10.1002/jbmr.4330

35. Burgess S, Thompson SG: Multivariable Mendelian randomization: the use of pleiotropic genetic variants to estimate causal effects. Am J Epidemiol 2015, 181(4):251-260 https://doi.org/https://doi.org/10.1093/aje/kwu283

36. Carter AR, Sanderson E, Hammerton G, Richmond RC, Davey Smith G, Heron J, Taylor AE, Davies NM, Howe LD: Mendelian randomisation for mediation analysis: current methods and challenges for implementation. Eur J Epidemiol 2021, 36(5):465-478 https://doi.org/https://doi.org/10.1007/s1 0654-021-00757-1

37. Marouli E, Del Greco MF, Astley CM, Yang J, Ahmad S, Berndt SI, Caulfield MJ, Evangelou E, McKnight B, Medina-Gomez C et al: Mendelian randomisation analyses find pulmonary factors mediate the effect of height on coronary artery disease. Commun Biol 2019, 2:119 https://doi.org/https:// doi.org/10.1038/s42003-019-0361-2, 1

38. Malik R, Chauhan G, Traylor M, Sargurupremraj M, Okada Y, Mishra A, Rutten-Jacobs L, Giese AK, van der Laan SW, Gretarsdottir S et al: Multiancestry genome-wide association study of 520,000 subjects identifies 32 loci associated with stroke and stroke subtypes. Nat Genet 2018, 50(4): 524-537 https://doi.org/https://doi.org/10.1038/s41588-018-0058-3

39. Malik R, Chauhan G, Traylor M, Sargurupremraj M, Okada Y, Mishra A, Rutten-Jacobs L, Giese AK, van der Laan SW, Gretarsdottir S et al: METASTROKE consortium. https://www.megastroke.org/ (2018)

40. Burgess S, Thompson DJ, Rees JMB, Day FR, Perry JR, Ong KK: Dissecting causal pathways using Mendelian randomization with summarized genetic data: application to age at menarche and risk of breast cancer. Genetics 2017, 207(2):481-487 https://doi.org/https://doi.org/10.1534/r.117.300191

41. Sanderson E, Davey Smith G, Windmeijer F, Bowden J: An examination of multivariable Mendelian randomization in the single-sample and twosample summary data settings. Int J Epidemiol 2019, 48(3):713-727 https:// doi.org/https://doi.org/10.1093/ije/dyy262

42. Rees JMB, Wood AM, Burgess S: Extending the MR-Egger method for multivariable Mendelian randomization to correct for both measured and unmeasured pleiotropy. Stat Med 2017, 36(29):4705-4718 https://doi.org/ https://doi.org/10.1002/sim.7492

43. Stout JC, Jernigan TL, Archibald SL, Salmon DP: Association of dementia severity with cortical gray matter and abnormal white matter volumes in dementia of the Alzheimer type. Arch Neurol 1996, 53(8):742-749 https:// doi.org/https://doi.org/10.1001/archneur.1996.00550080056013

44. Katsi V, Georgiopoulos G, Skafida A, Oikonomou D, Klettas D, Vemmos K, Tousoulis D: Noncardioembolic stroke in patients with atrial fibrillation. Angiology 2019, 70(4):299-304 https://doi.org/https://doi.org/10.1177/ 0003319718791711

45. Brodtmann A, Khlif MS, Egorova N, Veldsman M, Bird LJ, Werden E: Dynamic regional brain atrophy rates in the first year after ischemic stroke. Stroke 2020, 51(9):e183-e192 https://doi.org/https://doi.org/10.1161/strokeaha.120. 030256

46. Kirchhof P, Camm AJ, Goette A, Brandes A, Eckardt L, Elvan A, Fetsch T, van Gelder IC, Haase D, Haegeli LM, Hamann F, Heidbüchel H, Hindricks G, Kautzner J, Kuck KH, Mont L, Ng GA, Rekosz J, Schoen N, Schotten U, Suling A, Taggeselle J, Themistoclakis S, Vettorazzi E, Vardas P, Wegscheider K, Willems S, Crijns HJGM, Breithardt G: Early rhythm-control therapy in patients with atrial fibrillation. N Engl J Med 2020, 383(14):1305-1316 https:// doi.org/https://doi.org/10.1056/NEJMoa2019422

47. Kim D, Yang PS, Sung JH, Jang E, Yu HT, Kim TH, Uhm JS, Kim JY, Pak HN, Lee MH, Lip GYH, Joung B: Less dementia after catheter ablation for atrial fibrillation: a nationwide cohort study. Eur Heart J 2020, 41(47):4483-4493 https://doi.org/https://doi.org/10.1093/eurheartj/ehaa726 
48. Kuzniatsova N, Shantsila E, Lip GY: Atrial fibrillation: Blockade of the reninangiotensin system in atrial fibrillation. Nat Rev Cardiol 2010, 7(8):428-430 https://doi.org/https://doi.org/10.1038/nrcardio.2010.103

49. Charitakis $\mathrm{E}$, Walfridsson $\mathrm{H}$, Nylander $\mathrm{E}$, Alehagen U: Neurohormonal activation after atrial fibrillation initiation in patients eligible for catheter ablation: a randomized controlled study. J Am Heart Assoc 2016, 5(12) https://doi.org/https://doi.org/10.1161/jaha.116.003957,

50. Friedman Jl, Tang CY, de Haas HJ, Changchien L, Goliasch G, Dabas P, Wang $V$, Fayad ZA, Fuster V, Narula J: Brain imaging changes associated with risk factors for cardiovascular and cerebrovascular disease in asymptomatic patients. JACC Cardiovasc Imaging 2014, 7(10):1039-1053 https://doi.org/ https://doi.org/10.1016/j.jcmg.2014.06.014

\section{Publisher's Note}

Springer Nature remains neutral with regard to jurisdictional claims in published maps and institutional affiliations.

Ready to submit your research? Choose BMC and benefit from:

- fast, convenient online submission

- thorough peer review by experienced researchers in your field

- rapid publication on acceptance

- support for research data, including large and complex data types

- gold Open Access which fosters wider collaboration and increased citations

- maximum visibility for your research: over $100 \mathrm{M}$ website views per year

At $\mathrm{BMC}$, research is always in progress.

Learn more biomedcentral.com/submissions 